\title{
Tunable optical delay using parametric amplification in highly birefringent optical fibers
}

\author{
Nour Nasser, Gil Fanjoux, ${ }^{*}$ Eric Lantz, and Thibaut Sylvestre \\ Institut FEMTO-ST, Département d'Optique P. M. Duffieux, UMR CNRS-Université de Franche-Comté 6174, \\ Route de Gray 25030 Besançon Cedex, France \\ *Corresponding author: gil.fanjoux@univ-fcomte.fr
}

\begin{abstract}
We theoretically study parametric amplification in highly birefringent optical fibers and show that large tunable optical delay or advancement can be achieved via slow and fast light propagation. We provide a clear derivation of the formula for the optical delay that originates from the imaginary part of the parametric gain. We also perform numerical simulations in both normal and anomalous dispersion regimes. In the latter case, results show that large nanosecond optical delay could, in principle, be obtained at $1550 \mathrm{~nm}$ in a 1-km-long polarization-maintaining fiber. We further demonstrate that the optical delay and advancement rely on a group-velocity locking between the two cross-polarized signal and idler pulses.
\end{abstract}

\section{INTRODUCTION}

Slow and fast light (SFL) propagation in optical fibers continues to be a field of active research, motivated by applications to optical communications, such as tunable optical delay lines or enhanced all-optical signal processing [1-3]. In the past few years, SFL propagation has been demonstrated using optical parametric amplification (OPA) in optical fibers [4, This simple technique opens a new mean for optically tunable delay lines because they are fully compatible with high-bitrate communication digital data [5]. In addition to providing tunable optical delay, fiber-based parametric slow-light systems can operate over selectable and wide frequency bands, and simultaneously provide pulse advancement for the idler wave. Large delays and delay tuning ranges of the order of $160 \mathrm{ps}$ for a $70 \mathrm{ps}$ wide pulse have been achieved in a $2 \mathrm{~km}$ standard telecommunication fiber by using narrowband OPA assisted by the Raman gain [4]. It has also been shown numerically that standard telecommunication fibers can exhibit parametric gain and delay fluctuations due to polarization mode dispersion (PMD) []].

In this work, we propose taking advantage of the vector nature of the parametric processes in highly birefringent optical fibers to realize large optical delay or advancement. Vector OPA gain indeed gives the opportunity to realize unique gain bandwidths at unique wavelengths compared with scalar systems. The main consequence is that larger optical delay can be, in principle, achieved. More specifically, we show that a picosecond pulse propagating on the slow (fast) axis of a polarization-maintaining fiber (PMF) can be optically delayed (advanced) by using OPA with the pump wave polarized at $45^{\circ}$ of the birefringent axes. We provide a clear derivation of the formula for the optical delay that originates from the imaginary part of the parametric gain. Numerical simulations have been performed in both normal and anomalous dispersion regimes. For instance, our results show that large 900 ps optical delay can, in principle, be obtained at $1550 \mathrm{~nm}$ in a 1-km-long polarization-maintaining single-mode fiber and with a $3 \mathrm{~W}$ input pump power. We also investigate the tunability for the optical delay and show that it does not linearly depend on the pump power compared to conventional Brillouin or Raman slow-light systems [7-9]. We finally demonstrate that this vector SFL propagation leads to group-velocity matching between the cross-polarized signal and idler pulses, an effect analogous to the soliton trapping previously demonstrated in highly birefringent optical fibers [10].

\section{THEORY}

The parametric gain in highly birefringent fibers relies on a degenerate vector four-wave mixing process among three complex fields: $A_{p}(z, t), A_{s}(z, t)$, and $A_{i}(z, t)$, corresponding to the pump, signal, and idler wave amplitudes with angular frequencies $\omega_{p}, \omega_{s}$, and $\omega_{i}$, respectively, that satisfy the energy conservation law $2 \omega_{p}=\omega_{s}+\omega_{i}$. In the undepleted pump regime, the coupled nonlinear Schrödinger equations (CNLSEs) lead to the following coupled amplitude equations for the Stokes signal pulse polarized on the slow axis $x$ and the anti-Stokes idler pulse on the fast one as $y[11]$ :

$$
\begin{aligned}
& \frac{\partial A_{s x}}{\partial z}=i \frac{\gamma P}{3} A_{i y}^{*} \exp (-i \kappa z), \\
& \frac{\partial A_{i y}}{\partial z}=i \frac{\gamma P}{3} A_{s x}^{*} \exp (-i \kappa z),
\end{aligned}
$$

where $\kappa$ is the phase mismatch given by $\kappa=\beta_{2} \Omega^{2}+\delta \Omega+\gamma P$, with $\beta_{2}$ as the group-velocity dispersion (GVD) coefficient and $\Omega=\omega_{s}-\omega_{p}$ as the pump-signal frequency detuning. The parameter $\delta=\left(n_{x}-n_{y}\right) / c$ is the birefringence over the speed of light, $\gamma$ is the fiber nonlinear coefficient, and $P$ is the total pump power. Note that the stimulated Raman scattering effect is neglected in our model for the sake of clarity [12]. It must, however, be taken into account when the frequency detuning is close to the Raman frequency shift $(13.2 \mathrm{THz})$. We will present in Section 5 some numerical simulations showing an extra time delay due to the Raman contribution for $15 \mathrm{THz}$. 
Assuming the signal and idler amplitudes as $A_{s}(z)=$ $A_{s}(0) \exp \left(g_{s} z\right)$ and $A_{i}(z)=A_{i}(0) \exp \left(g_{i} z\right)$ and substituting into Eqs. (1) with only the pump and signal as initial conditions, one gets the following complex parameters $g_{s}$ and $g_{i}$ as

$$
\begin{aligned}
g_{s} & =\left(\frac{\gamma P}{3}\right)^{2} \frac{1}{g} \frac{\sinh (g z)}{\cosh (g z)+\frac{i \kappa}{2 g} \sinh (g z)}, \\
g_{i} & =g \frac{\cosh (g z)}{\sinh (g z)}-i \frac{\kappa}{2},
\end{aligned}
$$

where

$$
g=\sqrt{\left(\frac{\gamma P}{3}\right)^{2}-\left(\frac{\kappa}{2}\right)^{2}}
$$

is the parametric gain per unit length. It is important to stress here that the real parts of $g_{s}$ and $g_{i}$ refer to the parametric gain, whereas the imaginary part acts on the phase of the signal and idler and induces the so-called parametric SFL effect. From the imaginary parts, we can readily obtain the group index variation and the optical delays as []]

$$
\begin{aligned}
& \Delta n_{g}^{s, i}=c \frac{\partial \Im m\left(g_{s, i}\right)}{\partial \Omega}, \\
& \Delta t_{N L}^{s, i}=\int_{0}^{L} \frac{\Delta n_{g}^{s, i}(z)}{c} \mathrm{~d} z,
\end{aligned}
$$

where $L$ is the fiber length. Note that, in Eq. (3), $\Omega$ is negative (positive) for a down-frequency-shifted Stokes (anti-Stokes) signal and, thus, $\Delta t_{N L}^{s, i}$ is positive (negative) when the signal pulse is delayed (advanced). For large gain ( $g z \gg 1)$, one gets $\mathfrak{I} m\left(g_{s}\right)=\mathfrak{\Im} m\left(g_{i}\right)=-\kappa / 2$ and one can readily obtain an analytical expression for the optical delay as

$$
\Delta t_{N L}^{s}=-\Delta t_{N L}^{i}=-\left(\beta_{2} \Omega+\frac{\delta}{2}\right) L .
$$

Equation (5) shows that the signal and idler pulses experience opposite optical delay and advancement that mainly depend on the birefringence, the dispersion, and the fiber length. Compared with the scalar systems [4]], the vector optical delay also scales with the birefringence of the fiber and, thus, can be much larger. To show the pump power dependence of the optical delay, Eq. (5) can be rewritten by considering a signal frequency satisfying the phase-matching $\kappa=0$. It is given by

$$
\Delta t_{N L}^{s}=-\Delta t_{N L}^{i}=L \sqrt{\left(\frac{\delta}{2}\right)^{2}-\beta_{2} \gamma P}
$$

For small $\gamma P$, one can approximate $\Omega \simeq-\delta / \beta_{2}[\underline{13}]$ and, thus, the optical delay is merely given by $\Delta t_{N L}^{s}=\delta L / 2$. We will see in Section 3 that this simplest expression of the optical delay is in good agreement with numerical simulations of the CNLSE. Our analytical results are summarized and plotted in Figs. 1(a) and $1(\mathrm{~b})$. All parameters are listed in the caption of Fig. 1 and correspond to the case of a normally dispersive PMF single mode in the visible, similar to that used in Ref [10]. Numerical simulations for a pump in the $1550 \mathrm{~nm}$ band will be presented

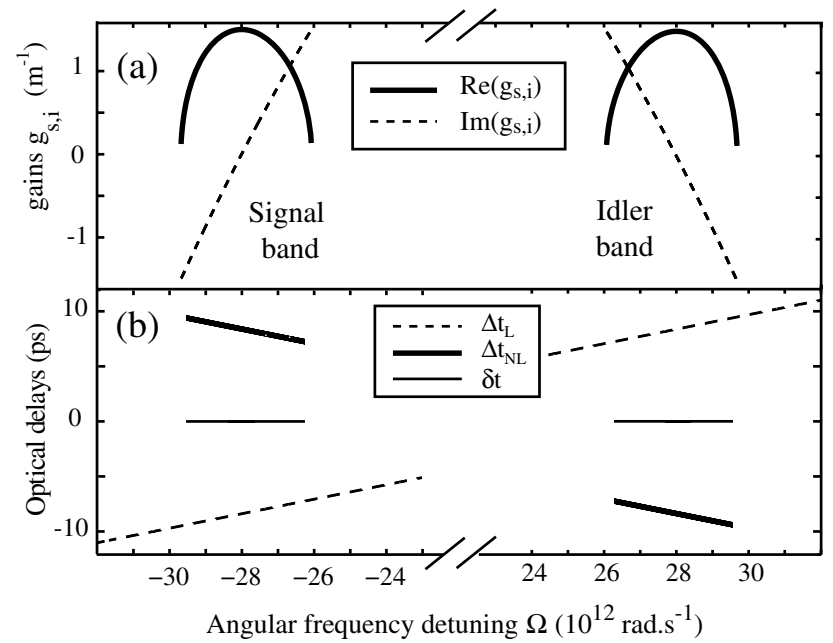

Fig. 1. (a) Evolution of the complex parameters $g_{s}$ and $g_{i}$ versus the angular frequency detuning $\Omega$ : the real part (parametric gain) as solid bell-shaped curves and the imaginary part as dashed curves. (b) Slow-light optical delay $\Delta t_{N L}$ (bold solid curve) and linear optical delay $\Delta t_{L}$ (dashed curve) due to birefringence and dispersion in the pump mean reference frame, and total delay $\delta t=\Delta t_{L}+\Delta t_{N L}$ (solid curve). Parameters are pump wavelength $\lambda_{p}=532 \mathrm{~nm}, \beta_{2}=$ $65.69 \times 10^{-27} \mathrm{~s}^{2} \cdot \mathrm{m}^{-1}, \quad \delta=2 \mathrm{ps} \cdot \mathrm{m}^{-1}, \quad \gamma=45 \times 10^{-3} \mathrm{~W}^{-1} \cdot \mathrm{m}^{-1}, \quad P=$ $100 \mathrm{~W}$, and $L=10 \mathrm{~m}$.

in Section 5. Figure 1(a) shows the real parts of $g_{s}$ and $g_{i}$ as solid bell-shaped curves and their imaginary parts as dashed curves versus the angular frequency detuning $\Omega$. Figure $\underline{1(b)}$ shows the optical delay $\Delta t_{N L}$ as bold solid curves generated by parametric gain. As it can be seen, for a peak pump power of $100 \mathrm{~W}$ and a fiber length of $10 \mathrm{~m}$ only, a signal pulse is optically delayed by about $8 \mathrm{ps}$ compared to the linear regime when the pump is off. Conversely, if the signal is located in the idler (anti-Stokes) band and polarized along the fast axis, it is advanced by the same amount, as shown by the solid curve for positive frequency detuning. It is noteworthy that the optical advancement is also due to a gain process, as the optical delay, and not to absorption, unlike the Brillouin or Raman fast-light processes [4]. Interestingly, the optical delay and advancement exactly compensate the linear walk-off due to the combined effects of birefringence and GVD in the pump mean reference frame $v_{g}=\left(v_{g}^{p x}+v_{g}^{p y}\right) / 2$. This walk-off is given by $\Delta t_{L}^{s}=-\Delta t_{L}^{i}=\left(\beta_{2} \Omega+\frac{\delta}{2}\right) L$ and is plotted in Fig. 1(b) as a dashed curve for comparison. The resulting total delay $\delta t=\Delta t_{N L}+\Delta t_{L}$ is also plotted in Fig. 1(b) as a thin solid curve. As it can be seen, it is remarkably zero and flat all over the parametric gain band and, thus, the signal and idler propagate with the same group velocity in spite of the high birefringence. This means that the parametric interaction and the induced SFL propagation lead to group-velocity locking of the signal and idler pulses, in a way analogous to the soliton trapping effect previously observed in PMFs in the framework of modulation instability [10].

\section{NUMERICAL SIMULATIONS: NORMAL DISPERSION}

To verify our analytical predictions, we have performed some numerical simulations of the CNLSEs in a highly birefringent fiber that is normally dispersive and single mode in the visible region. As input conditions, we considered a $300 \mathrm{ps}$ square pump pulse at a wavelength of $532 \mathrm{~nm}$ and polarized at $45^{\circ}$ 
of the neutral axes of the fiber [10]. The signal pulse duration was set to $10 \mathrm{ps}$ (Gaussian, full width at half-maximum) and its wavelength was fixed at $536.26 \mathrm{~nm}$, i.e., to the peak of the gain band shown in Fig. 1(a). Others parameters are the same as in the caption of Fig. 1. Figure 2(a) shows the signal pulse at the output of the birefringent fiber in normalized units in the linear (dashed curve) and nonlinear (solid curve) regimes. Further comparison between both regimes shows that the signal pulse is optically delayed by nearly 8 ps without significant pulse distortion. The idler pulse generated by OPA is also plotted as a dotted curve. We can see that it is almost synchronized with the signal pulse due to the group-velocity locking effect discussed in Section 2 . Note that there still remains a weak residual delay of $0.6 \mathrm{ps}$ between the signal and the idler at the fiber output because of the transient weak parametric gain regime. This residual delay can be also viewed in Fig. 2(b), which shows the optical delay versus the propagation distance for the signal in the linear (dashed) and nonlinear (solid) regimes and for the idler (dotted). The optical delay per meter is about $0.8 \mathrm{ps} \cdot \mathrm{m}^{-1}$, which corresponds to roughly half of the birefringence parameter $\delta=2 \mathrm{ps} \cdot \mathrm{m}^{-1}$, as previously predicted by our simple analytical expression. Figure 2(b) also shows that the optical delay is proportional to the fiber length, as expected from theory.

\section{TUNABILITY OF THE OPTICAL DELAY}

In this section we investigate the tunability of the parametric optical delay when the pump power is varied while maintaining the signal wavelength at the peak of the gain band. Equation (6) shows that the delay does not linearly depend on the pump power, unlike the other fiber-based Brillouin or Raman slow-light systems [3]. This can be explained by the fact that the parametric gain spectrum broadens and shifts when increasing the pump power and the resulting optical delay thus remains quasi-constant in the steady-state gain regime. This is illustrated in Fig. 3(a), which shows the optical delay (black squares) and the output signal duration (open circles) versus the pump power for a tunable phase-matched signal. For more details, we have also plotted on Fig. 3(b) the optical delay versus the propagation distance for four different pump
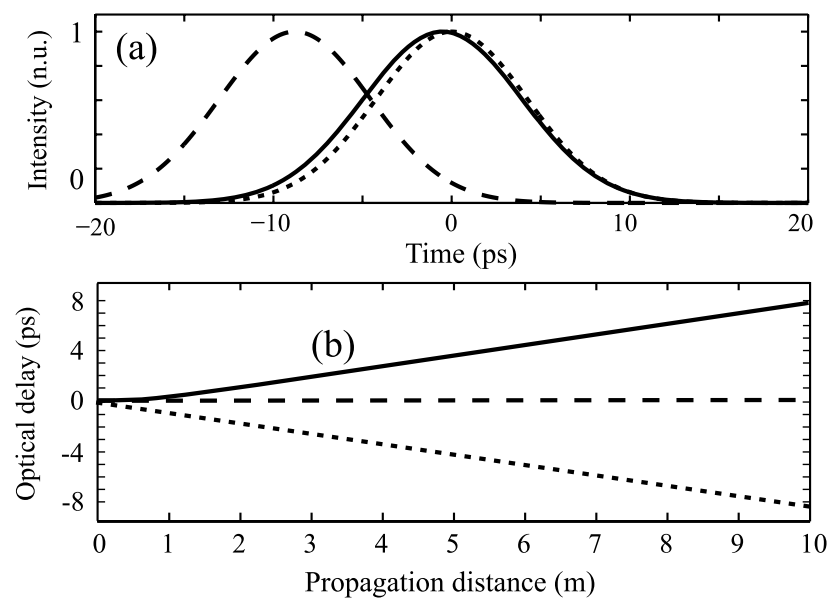

Fig. 2. Numerical simulations. (a) Output intensity pulse profiles in normalized units and (b) optical delay versus the propagation distance, for the signal and for the spontaneously generated idler in the nonlinear regime as solid and dotted curves, respectively. For comparison, the dashed curve indicates the signal position in the linear regime. Parameters are the same as in Fig. 1.
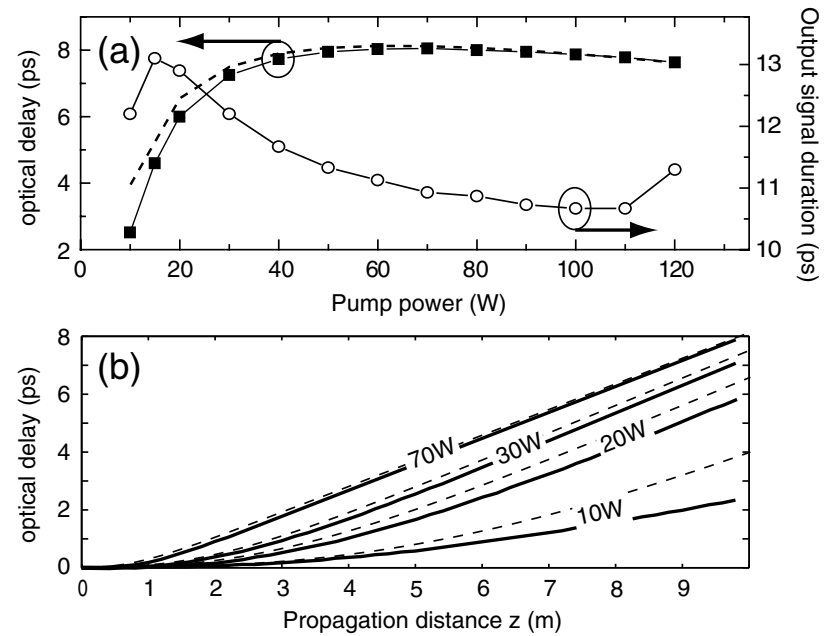

Fig. 3. (a) Optical delay (left, squares) and pulse duration (right, open circles) versus the pump power and for a fiber length of $10 \mathrm{~m}$. (b) Optical delay versus the propagation distance for four different pump powers. Dashed curves in both frames show comparison with Eq. (4).

powers. The delay predicted by Eq. (4) is also plotted as dashed curves and shows rather good agreement with numerical simulations. As can be seen in Fig. 3(a), there is a transient regime for the optical delay that rapidly increases from $2 \mathrm{ps}$ to reach its maximum value of $8 \mathrm{ps}$ in the steady-state gain regime for pump power of $70 \mathrm{~W}$. Then it saturates and slightly decreases as a function of the pump power, as expected from the analytical expression of Eq. (6). This is due to the fact that the pump-signal detuning $\Omega$ reduces as the signal is maintained at the peak of the gain band, leading to the decrease of optical delay, as predicted by Eq. (5). Note that, in the transient regime, the pulse broadens from 10 to $13 \mathrm{ps}$, whereas it remains nearly the same for high power. Thus, we can conclude from Fig. 3 that there is a pump power range for which the optical delay is maximum and the pulse suffers no significant broadening. In Fig. 3, this corresponds to a pump power range between 60 and $110 \mathrm{~W}$. To get better insight, Fig. 4 illustrates a color plot of the parametric optical delay in function of both the pump power $P$ and the fiber length $L$ obtained from numerical simulations of the CNLSEs. For comparison, the results of analytical predictions from Eq. (4) are superimposed as mesh curves. It is clear from Fig. $\underline{4}$ that the optical

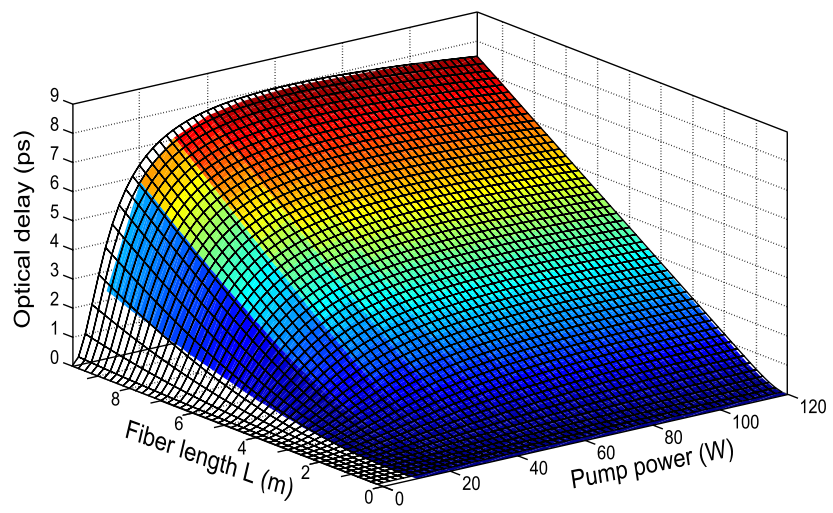

Fig. 4. (Color online) Parametric optical delay as a function of both the pump power and the fiber length. Comparison of numerical simulations from CNLSEs (color map) with analytical predictions from Eq. (4) (mesh black curves). 
delay rapidly saturates with the pump power independently of the fiber distance. However, it linearly increases versus the propagation distance as predicted by Eq. (6), indicating that large optical delay can, in principle, be obtained with a long fiber length.

\section{ANOMALOUS DISPERSION CASE}

In view of potential applications to practical telecommunication systems, we have additionally performed some numerical simulations in a 1-km-long PMF and for a signal wavelength at $1.5 \mu \mathrm{m}$. To be optically delayed by OPA the signal pulse must be polarized along the slow axis and located in the anti-Stokes gain band owing to the phase-matching condition. This is due to the fact that the signal propagates in the anomalous dispersion regime of the fiber, unlike the previous case in the visible. We have numerically calculated from Eq. (4) the parametric optical delay in function of both the pump power and the fiber length for a birefringence parameter of $\delta=2 \mathrm{ps} \cdot \mathrm{m}^{-1}$, for a nonlinear parameter of $\gamma=5 \times 10^{-3} \mathrm{~W}^{-1} \cdot \mathrm{m}^{-1}$, for a GVD parameter of $\beta_{2}=-60 \mathrm{ps} \cdot \mathrm{m}^{-1}$, and for a square pump pulse with a duration of $10 \mathrm{~ns}$. Phase matching for vector OPA is thus achieved for a pump wavelength of $1570 \mathrm{~nm}$. Figure $\underline{5}$ shows the results of our analytical predictions given by Eq. (4). The pump power was varied from 0 to $10 \mathrm{~W}$ and the fiber length from 0 to $2 \mathrm{~km}$. Figure 5 first shows that the optical delay can reach a large value up to $1800 \mathrm{ps}$ at a propagation distance of $2 \mathrm{~km}$ and for a pump power of $10 \mathrm{~W}$. Therefore, it is noteworthy that our system can deliver more than 10 times larger delays than those obtained from Ramanassisted OPA [4] with similar pump power and fiber length. Another advantage is that these delays can be obtained regardless of the signal wavelength against the zero-dispersion wavelength. In addition, these large delays depend on the birefringence of the fiber and can still be improved by using, for example, highly birefringent photonic crystal fibers [14].

Shifting the signal wavelength from the visible to the IR band implies some important changes in the optical delay line. First of all, the decrease of the $\gamma$ nonlinear coefficient (with a ratio of 9 here) leads to the reduction of the gain in the same proportion. The gain bandwidth is significantly narrowed (with a ratio of 30 here), leading to a high sensitivity to experimental parameter variations, such as birefringence, dispersion, and pump power. Correspondingly, longer signal pulse durations than for the visible case are required to avoid

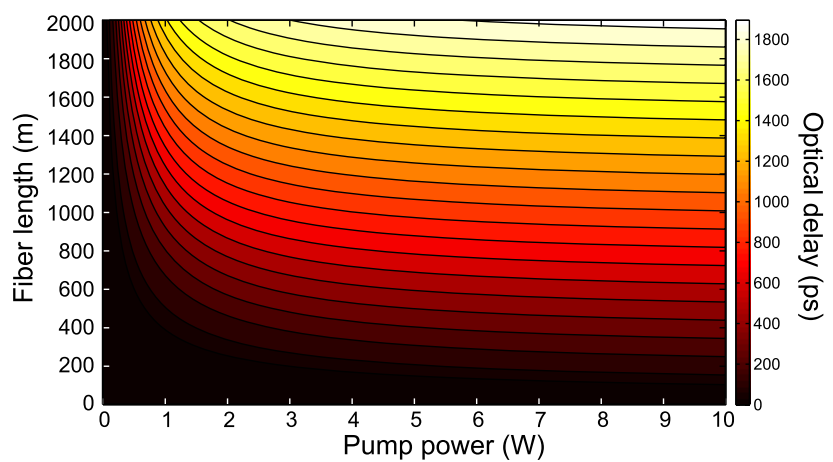

Fig. 5. (Color online) Optical delay (color bar) generated by vector optical parametric amplification in a polarization-maintaining singlemode fiber versus both the pump power and the fiber length. Parameters are: signal wavelength $\lambda_{s}=1550 \mathrm{~nm}, \beta_{2}=-60 \times 10^{-27} \mathrm{~s}^{2} \cdot \mathrm{m}^{-1}$, $\delta=2 \mathrm{ps} \cdot \mathrm{m}^{-1}$, and $\gamma=5 \times 10^{-3} \mathrm{~W}^{-1} \cdot \mathrm{m}^{-1}$.
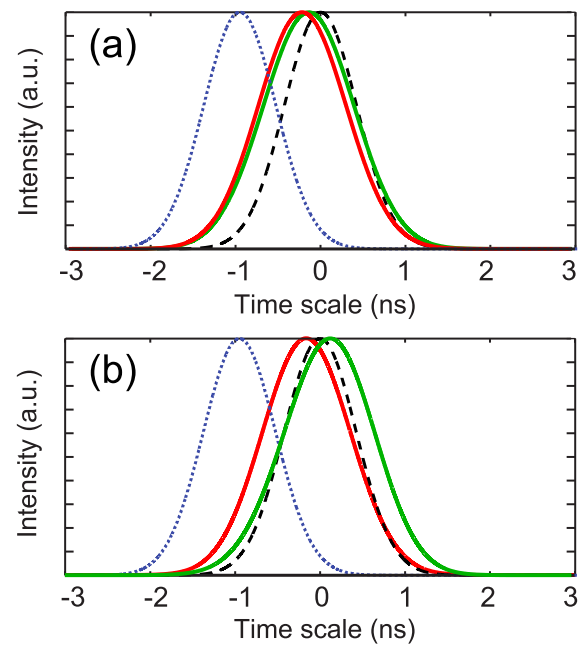

Fig. 6. (Color online) Comparison between output pulses with and without the Raman scattering for two angular frequency detuning $\Omega$ showing the beneficial effect of Raman contribution to the parametric optical delay. (a) $\Omega / 2 \pi=3.5 \mathrm{THz}$ and (b) $\Omega / 2 \pi=15 \mathrm{THz}$. Dashed curves, input signal pulse; dotted curves, output signal in the linear regime; solid red (left) and green (right) curves, output signal in the nonlinear regime without and with Raman contribution, respectively.

pulse broadening due to the parametric gain filtering process, as we will see in Section 6 .

It is important to note that the Raman effect is known to influence the optical delay induced by OPA $[4,15]$. The real and imaginary parts of the Raman susceptibility must be taken into account in the model when the frequency detuning is close to the Raman frequency shift $(13.2 \mathrm{THz})$. We have included the Raman effect in our CNLSE-based numerical simulations. Figures 6 show the results of these numerical simulations for two different detunings: 5.3 and $15 \mathrm{THz}$. As can be seen, for $15 \mathrm{THz}$, the Raman effect significantly increases the optical delay for the pulse signal by $35 \%$ (1.1 ns instead of $800 \mathrm{ps}$ without the Raman effect) whereas it slightly increases for $5.3 \mathrm{THz}$ by $5 \%$ ( $840 \mathrm{~ns}$ instead of 800 ps without the Raman effect). This additional optical delay actually results from the Raman contribution to the Kerr effect associated with the imaginary part of the Raman susceptibility. Including the Raman contribution to the Kerr effect in our analytical study is, however, not very simple. Raman-assisted vector OPA requires a new detailed analytical and numerical study that will be done in the near future.

\section{OPTICAL DELAY LIMITATIONS}

The first limitation of the optical delay range generated by vector OPA is the parametric gain limit. A $70 \mathrm{~dB}$ gain actually represents the upper limit [16]. Figure 7 shows the evolution of the parametric gain in logarithm scale as a function of both the pump power and the fiber length. Further comparison between Fig. $\underline{5}$ and Fig. 7 highlights the significant reduction of the delay range by the gain limitation. In addition, it is noteworthy that pump depletion generally occurs before the upper gain limit. The pump depletion depends on the input pump and signal powers and on the fiber length. In addition to the optical delay saturation, pump depletion also leads to a signal pulse distortion.

The second limitation of the optical range corresponds to the signal pulse broadening due to the narrow gain spectrum, 


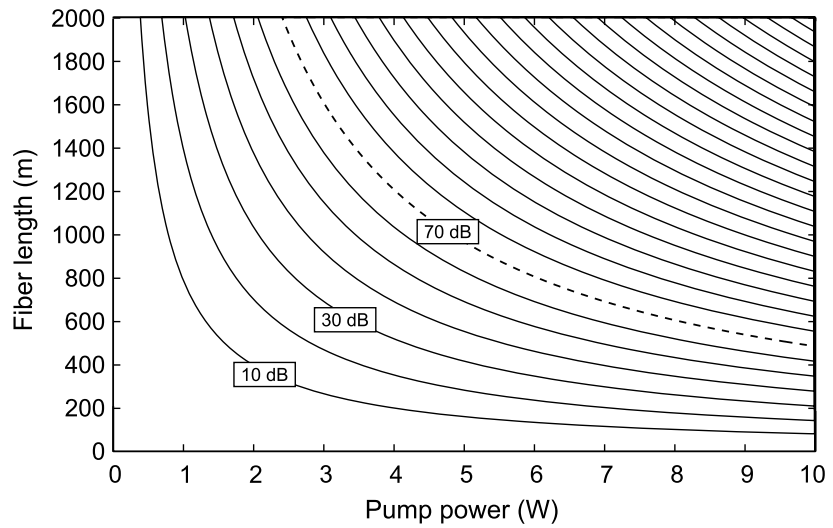

Fig. 7. Analytical prediction for the gain (in decibels) in function of the pump power and the fiber length ( $10 \mathrm{~dB}$ per curve).

as previously shown in Fig. 3(a). This gain-dependent pulse broadening depends on the input signal pulse duration $T_{0}$, the propagation length $L$, and the pump power. More specifically, the broadening ratio for the signal pulse can be estimated from the following equation [17]:

$$
R=\frac{T}{T_{0}}=\sqrt{1+\frac{G L}{\left(\Delta \Omega T_{0}\right)^{2}}},
$$

with $G$ as the peak of the gain (in $\mathrm{m}^{-1}$ ) and $\Delta \Omega$ as the gain bandwidth for half-width at half-maximum. Note that we have neglected in Eq. (7) the GVD-induced pulse broadening that is negligible for nanosecond pulses as in our case. Figure $\underline{8}$ shows the evolution of the optical delay and the broadening coefficient versus the parametric gain (in decibels) and fiber length. The input signal pulse duration is $1 \mathrm{~ns}$. As can be seen, the optical delay and the broadening coefficient strongly vary with the gain. There is a trade-off between the maximum optical delay and the pulse broadening factor. As the latter should have to be limited, the optimal delay line would be an optical fiber system of $1 \mathrm{~km}$ generating $0.9 \mathrm{~ns}$ optical delay. In addition, the weak gain region (below $20 \mathrm{~dB}$ typically) must be avoided in order to prevent large pulse broadening, as shown by the dashed curves in Fig. 8. By comparing these results with those found in Ref. [4], it is noteworthy that the optical delays generated by vector OPA gain are 1 order of magnitude larger than those obtained by Raman-assisted

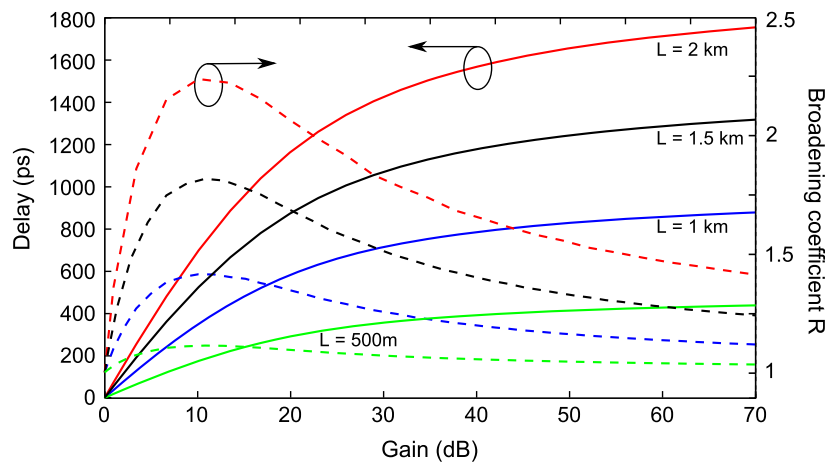

Fig. 8. (Color online) Optical delay (right, solid curves) and broadening coefficient (left, dashed curves) versus the parametric gain in decibels and for propagation distances ranging from $500 \mathrm{~m}$ (green curves) to $2 \mathrm{~km}$ (red curves). The blue curves correspond to $1 \mathrm{~km}$ of propagation. The input signal pulse duration is $1 \mathrm{~ns}$.
OPA with similar parameters, which may represent a significant improvement.

\section{CONCLUSION}

In conclusion, we have proposed and theoretically demonstrated that optical parametric amplification in highly birefringent fiber can be advantageously used to generate large pulse optical delay or advancement. We have provided a derivation for the optical delay that depends on the fiber birefringence and we have shown that this effect leads to group-velocity locking between the cross-polarized signal and idler pulses. As parametric amplification is analogous to the induced modulation instability process, we expect that large optical delay could also be achieved by using polarization modulation instability in weakly birefringent fibers [18].

\section{ACKNOWLEDGMENTS}

The authors acknowledge the Conseil Régional de FrancheComté for financial support. Computations have been performed on the supercomputer facilities of the Mésocentre de calcul de Franche-Comté.

\section{REFERENCES}

1. J. B. Khurgin and R. S. Tucker eds., Slow Light: Science and Applications (CRC Press, 2009).

2. R. Boyd, "Slow and fast light: fundamentals and applications," J. Mod. Opt. 56, 1908-1915 (2009).

3. L. Thévenaz, "Slow and fast light in optical fibres," Nat. Photon. 2, 474-481 (2008).

4. D. Dahan and G. Eisenstein, "Tunable all optical delay via slow and fast light propagation in a Raman assisted fiber optical parametric amplifier: a route to all optical buffering," Opt. Express 13, 6234-6249 (2005).

5. E. Shumakher, A. Willinger, R. Blit, D. Dahan, and G. Eisenstein, "Large tunable delay with low distortion of $10 \mathrm{Gbit} / \mathrm{s}$ data in a slow light system based on narrow band fiber parametric amplification," Opt. Express 14, 8540-8545 (2006).

6. L. Schenato, M. Santagiustina, and C. G. Someda, "Fundamental and random birefringence limitations to delay in slow light fiber parametric amplification,” J. Lightwave Technol. 26, 3721-3726 (2008).

7. Y. Okawachi, M. S. Bigelow, J. E. Sharping, Z. Zhu, A. Schweinsberg, D. J. Gauthier, R. W. Boyd, and A. L. Gaeta, "Tunable all-optical delays via Brillouin slow light in an optical fiber," Phys. Rev. Lett. 94, 153902 (2005).

8. K. Y. Song, M. G. Herráez, and L. Thévenaz, "Observation of pulse delaying and advancement in optical fibers using stimulated Brillouin scattering," Opt. Express 13, 82-88 (2005).

9. J. E. Sharping, Y. Okawachi, and A. L. Gaeta, "Wide bandwidth slow light using a Raman fiber amplifier," Opt. Express 13, 6092-6098 (2005).

10. E. Seve, G. Millot, S. Wabnitz, T. Sylvestre, and H. Maillotte, "Generation of vector dark-soliton trains by induced modulational instability in a highly birefringent fiber," J. Opt. Soc. Am. B 16, 1642-1650 (1999).

11. R. H. Stolen and J. E. Bjorkholm, "Parametric amplification and frequency conversion in optical fibers," IEEE J. Quantum Electron. 18, 1062-1072 (1982).

12. G. P. Agrawal, Nonlinear Fiber Optics, 4th ed. (Academic, 2007).

13. A. T. Nguyen, K. P. Huy, E. Brainis, P. Mergo, J. Wojcik, T. Nasilowski, J. Van Erps, H. Thienpont, and S. Massar, "Enhanced cross phase modulation instability in birefringent photonic crystal fibers in the anomalous dispersion regime," Opt. Express 14, 8290-8297 (2006).

14. A. Ortigosa-Blanch, J. C. Knight, W. J. Wadsworth, J. Arriaga, B. J. Mangan, T. A. Birks, and P. St. J. Russell, "Highly birefringent photonic crystal fibers," Opt. Lett. 25, 1325-1327 (2000). 
15. A. Willinger, E. Shumakher, and G. Eisenstein, "On the roles of polarization and Raman-assisted phase matching in narrowband fiber parametric amplifiers," J. Lightwave Technol. 26, 2260 2268 (2008).

16. T. Torounidis, P. A. Andrekson, and B.-E. Olsson, "Fiber-optical parametric amplifier with $70 \mathrm{~dB}$ gain," IEEE Photon. Technol. Lett. 18, 1194-1196 (2006).
17. R. W. Boyd, D. J. Gauthier, A. L. Gaeta, and A. E. Willner, "Maximum time delay achievable on propagation through a slow-light medium," Phys. Rev. A 71, 023801 (2005).

18. S. G. Murdoch, R. Leonhardt, and J. D. Harvey, "Polarization modulation instability in weakly birefringent fibers," Opt. Lett. 20, 866-868 (1995). 